\title{
Editorial
}

\section{Fbxo45 joins the 'Par-4'ty in controlling apoptosis of cancer cells}

\author{
Z Wang ${ }^{1,2}$ and W Wei ${ }^{\star \star 2}$ \\ Cell Death and Differentiation (2014) 21, 1508-1510; doi:10.1038/cdd.2014.104
}

Ubiquitination by the ubiquitin proteasome system (UPS) has been characterized as a critical type of post-translational modification, which governs multiple important cellular processes including cell survival, apoptosis, migration, invasion, and metastasis primarily via regulating the abundance of key cellular proteins. ${ }^{1}$ This ubiquitination process is exerted by an enzymatic cascade including the ubiquitin-activating enzyme (E1), the ubiquitin-conjugating enzyme (E2), and ubiquitinprotein ligases (E3). ${ }^{2}$ So far, the E3 ubiquitin ligases responsible for target specificity have been categorized into several classes based on their special domains, such as the RING (really interesting new gene)-domain and the HECT (homologous to the E6-AP carboxyl terminus)-domain type of E3 ligases. ${ }^{3}$ Among them, the Skp1-Cullin1-F-box complex (SCF) subfamily is one of the best characterized multicomponent RING-type E3 ligases. Specifically, the SCF complex contains four components: Skp1, Rbx1, Cullin1, and F-box protein. ${ }^{4}$ Around 70 putative F-box proteins including Fbxo45 have been identified in the human genome. ${ }^{5}$ However, besides the well-studied F-box proteins such as Skp2 (S-phase kinase-associated protein 2$)^{6}$ or Fbw7 (F-box and WD repeat domain-containing 7$),{ }^{7}$ the physiological function and substrates of many F-box proteins including Fbxo45 remain largely elusive.

Among these under-studied F-box proteins, Fbxo45 has been reported to be involved in neural development and tumorigenesis. $^{8,9}$ Fbxo45 gene was first reported as an estrogen-induced gene that consists of several estrogen receptor-binding consensus sequences. ${ }^{10}$ Moreover, 17betaestradiol treatment induced Fbxo45 expression in MCF-7 breast cancer cells. ${ }^{10}$ Interestingly, the alteration of Fbxo45 mRNA levels was not observed in male zebrafish after 17alpha-ethinylestradiol treatment, ${ }^{11}$ suggesting that further investigation is required to explore the relationship between Fbxo45 and estrogen in different species. Notably, Fbxo45 was identified as a new attractive candidate gene for schizophrenia. $^{12}$ Intriguingly, unlike other F-box proteins, Fbxo45 forms an atypical ubiquitin ligase complex that consists of Skp1 and PAM (protein associated with Myc). ${ }^{8}$ Additionally, Fbxo45 contains a SPRY domain at its c-terminus that mediates the interaction with most of its substrates. ${ }^{13}$ Fbxo45 knockout mice are embryonic lethal due to abnormal innervations of the diaphragm, impaired synapse formation, and aberrant development of axon fiber tracts in the brain, suggesting that Fbxo45 might play a critical role in neural development. ${ }^{8}$ Consistently, it has been demonstrated that Fbxo45 regulates neurotransmission by modulating degradation of a synaptic vesicle-priming factor, Munc13-1, at the synapse. ${ }^{14}$ Recently, Fbxo45 was also found to mediate proteasome-dependent degradation of the transcription factor p73, a well-characterized tumor suppressor that plays a crucial role in cell cycle and apoptosis. ${ }^{9}$ Specifically, depletion of endogenous Fbxo45 stabilizes p73 and sensitizes breast cancer cell line to doxorubicin-induced cell death, indicating that Fbxo45 could exert oncogenic function. ${ }^{9}$ Although a line of evidence reveals the oncogenic role of Fbxo45, the exact molecular mechanisms underlying Fbxo45-mediated carcinogenesis are largely unclear.

In this issue of $C D D$, Chen et al. ${ }^{15}$ seek to understand the critical role and molecular mechanisms underlying Fbxo45mediated ubiquitination of the Par-4 (prostate apoptosis response protein 4) tumor suppressor to regulate cancer cell survival (Figure 1). It has been well accepted that Par-4 functions as a tumor suppressor largely through induction of cellular apoptosis of cancer cells. ${ }^{16}$ It is important to note that Par-4 triggers apoptosis when it translocates to the nucleus from the cytoplasm. ${ }^{16}$ Given that Par-4 induces apoptosis, Par-4 knockout mice develop tumors in multiple organs such as the liver, lung, and endometrium. Consistently, low levels of Par-4 expression are associated with poor prognosis in breast cancer, pancreatic cancer, and lung cancer, ${ }^{17}$ further advocating its tumor suppressor role. Accumulating evidence suggests that Par-4 suppresses tumorigenesis via translocating to the nucleus and subsequently inhibiting NF- $\kappa \mathrm{B}$ (nuclear factor- $\kappa \mathrm{B}$ ), Akt, PKC $\delta$ (protein kinase $\mathrm{C} \delta$ ), and TOPO1 (topoisomerase-1). ${ }^{18,19}$ However, the detailed mechanism by which Par-4 induces apoptosis in cancer has not been formally addressed.

Chen et al. ${ }^{15}$ identified Par-4 as a novel Fbxo45 interacting protein using immunopurification coupled with an unbiased mass spectrometry approach. The authors further confirmed the binding of Fbxo45 and Par-4 by immunoprecipitation and western blot analyses. Moreover, this group detected only Fbxo45, but not other F-box proteins such as $\beta$-TRCP

\footnotetext{
${ }^{1}$ The Cyrus Tang Hematology Center and Collaborative Innovation Center of Hematology, Jiangsu Institute of Hematology, The First Affiliated Hospital, Soochow University, Suzhou 215123, People's Republic of China and ${ }^{2}$ Department of Pathology, Beth Israel Deaconess Medical Center, Harvard Medical School, Boston, MA 02215, USA ${ }^{*}$ Corresponding author: W Wei, Department of Pathology, Beth Israel Deaconess Medical Center, Harvard Medical School, 330 Brookline Avenue, Boston, MA 02215, USA. Tel: +1 617735 2495; Fax: +1 617735 2480; E-mail: wwei2@ bidmc.harvard.edu
} 


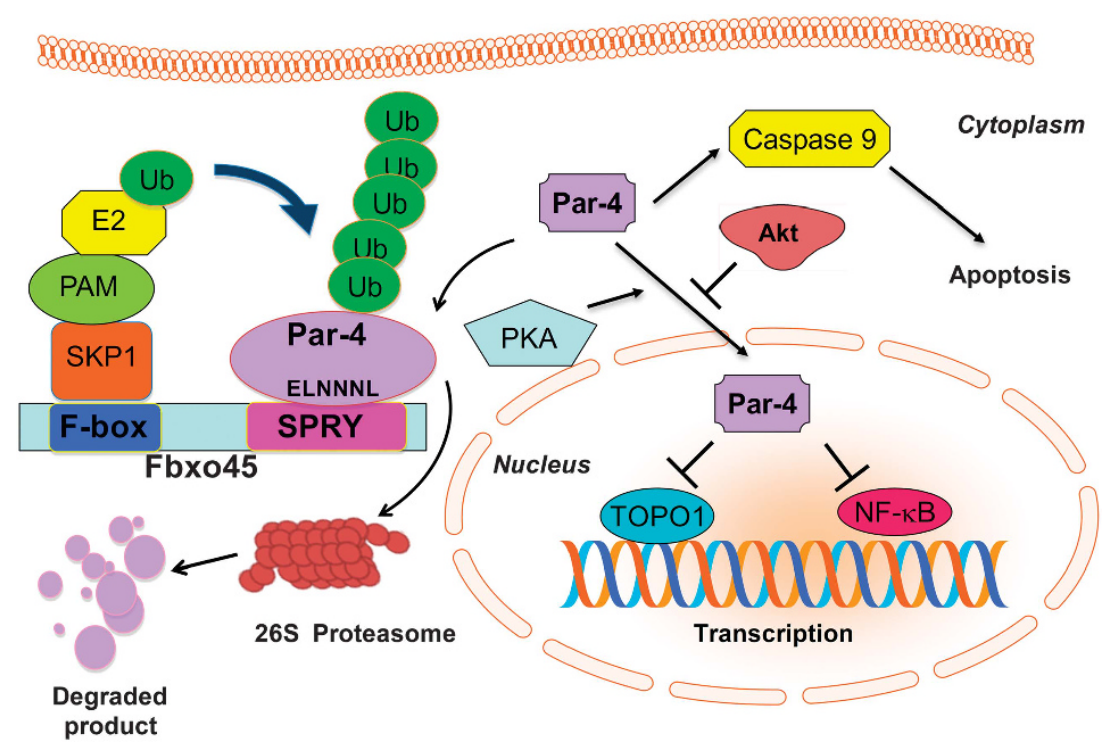

Figure 1 Role of Fbxo45 in regulating apoptosis by promoting the degradation of Par-4 in cancer cells. In carcinoma cells, Par-4 triggers cellular apoptosis through activation of caspase 9. Moreover, Par-4 suppresses tumorigenesis via translocating to the nucleus, an event that is promoted by PKA-dependent phosphorylation, and subsequently inhibits NF- $\kappa$ B and TOPO1 signaling. The tumor suppressor functions of Par-4 can be compromised through different oncogenic pathways. For example, Akt could inhibit Par-4 translocation to the nucleus by phosphorylating Par-4. Furthermore, Fbxo45 interacts with Par-4 in the cytoplasm and mediates its ubiquitination and subsequent proteasomal degradation, leading to cancer cell survival

(beta transducin repeat containing protein) and Skp2, to co-immunoprecipitate with endogenous Par-4. ${ }^{15}$ Furthermore, Fbxo45 bound Skp1 and PAM, but not Cullin 1 and Rbx1, to form an atypical form of SCF E3 ligase complex. Notably, SPRY domain mediates the interaction between Fbxo45 and Par-4 in the cytoplasm. With a combined biochemical and cellular approach, Chen et al. ${ }^{15}$ further identified the Par-4 tumor suppressor as a novel Fbxo45 substrate and further demonstrated that the specific interaction between the SPRY domain of Fbxo45 and the ELNNNL motif of Par-4 is critical for Fbxo45-mediated ubiquitination and subsequent degradation of Par-4. Moreover, their results demonstrated that Fbxo45 promotes the Par-4 degradation in the cytoplasm. Consistent with this finding, aberrant translocation to the nucleus protects Par-4 from being degraded by Fbxo45.

The authors also explored whether Fbxo45-mediated degradation of Par-4 is involved in modulating cellular apoptosis in cancer cells. To address this critical question, this group detected the sensitivity of cancer cells expressing the Par-4 wild-type (WT) or Par-4 (3A) mutant to staurosporine-induced apoptosis. ${ }^{15}$ To this end, they reported that cells expressing Par-4 (3A) mutant have a significant increase in the number of apoptotic cells compared to the cells with Par-4 (WT). ${ }^{15}$ Consistently, TUNEL and western blot analyses for cleaved PARP-1 as additional apoptotic markers confirmed that Par-4 (3A) mutant promoted staurosporineinduced apoptosis. In support of this conclusion, they further showed that misregulated degradation of Par-4 by depletion of Fbxo45 or mutating the degron could also trigger cellular apoptosis. $^{15}$

Although this interesting study shed important lights on the roles of Fbxo45 in regulation of apoptosis in cancer cells, a couple of critical questions still need to be answered in follow- up studies. For example, to validate the physiological role of Fbxo45 in vivo, Fbxo45 transgenic mice should be generated to address whether Fbxo45 facilitates tumorigenesis in part through Par-4 degradation. It also remains unclear whether Fbxo45 mediates degradation of apoptotic proteins other than Par-4 to control tumorigenesis. In this regard, Par-4 has been reported to inhibit tumor growth and metastasis progression. ${ }^{20}$ Thus it should be further addressed whether elevated Fbxo45 expression could facilitate tumor metastasis in part via promoting Par-4 degradation. Furthermore, reduced Par-4 expression is correlated to poor prognosis in cancer patients; thus, it is interesting to explore whether Fbxo45 overexpression could also be used as a biomarker of prognosis for human cancers. A more important question is whether tissue-specific Fbxo45 knockout or knockin mouse models could confirm its oncogenic role in tumorigenesis. Without any doubt, further studies are warranted to determine whether Fbxo45 could represent a prospective therapeutic target for human malignancies.

\section{Conflict of Interest}

The authors declare no conflict of interest.

Acknowledgements. This work was supported by a grant from NSFC (81172087) and the priority academic program development of Jiangsu highereducation institutions. This work was supported in part by the NIH grants to WW (GM089763, GM094777, and CA177910). WW is an ACS research scholar and a LLS research scholar.

1. Wang Z et al. Nat Rev Cancer 2014; 14: 233-247.

2. Skaar JR, Pagan JK, Pagano M. Nat Rev Mol Cell Biol 2013; 14: 369-381.

3. Nakayama KI, Nakayama K. Nat Rev Cancer 2006; 6: 369-381.

4. Lipkowitz S, Weissman AM. Nat Rev Cancer 2011; 11: 629-643.

5. Cardozo T, Pagano M. Nat Rev Mol Cell Biol 2004; 5: 739-751.

6. Frescas D, Pagano M. Nat Rev Cancer 2008; 8: 438-449.

7. Welcker M, Clurman BE. Nat Rev Cancer 2008; 8: 83-93. 
8. Saiga T et al. Mol Cell Biol 2009; 29: 3529-3543.

9. Peschiaroli A et al. Oncogene 2009; 28: 3157-3166.

10. Yoshida K. Oncol Rep 2005; 14: 531-535.

11. Zheng $\mathrm{H}$ et al. J Environ Sci (China) 2011; 23: 664-670.

12. Wang C et al. Schizophr Res 2014; e-pub ahead of print 27 May 2014; doi:10.1016/ j.schres.2014.04.032

13. Jin J et al. Genes Dev 2004; 18: 2573-2580.
14. Tada $\mathrm{H}$ et al. J Biol Chem 2010; 285: 3840-3849.

15. Chen X et al. Cell Death Differ 2014; 21: 1535-1545.

16. Qinan W, Ling Z, Bing C. Expert Opin Ther Targets 2014; 1-11.

17. Irby RB, Kline CL. Expert Opin Ther Targets 2013; 17: 77-87.

18. Fernandez-Marcos PJ et al. Proc Natl Acad Sci USA 2009; 106: 12962-12967.

19. Joshi J et al. EMBO J 2008; 27: 2181-2193.

20. Zhao Y et al. Cancer Biol Ther 2011; 12: 152-157. 